\title{
Hypertensive disorders in pregnancy and maternal and neonatal outcomes in Haiti: the importance of surveillance and data collection
}

Matthew Bridwell ${ }^{1 *}$ (D) Endang Handzel ${ }^{1}$, Michelle Hynes ${ }^{1}$, Reginald Jean-Louis ${ }^{1}$, David Fitter ${ }^{1}$, Carol Hogue ${ }^{2}$, Reynold Grand-Pierre ${ }^{3}$, Hedwige Pierre ${ }^{4}$ and Bradley Pearce ${ }^{2}$

\begin{abstract}
Background: This study aims to determine reported prevalence of hypertensive disorders in pregnancy (HDP) and maternal and neonatal outcomes associated with these disorders among women delivering at selected hospitals across Haiti.

Methods: A retrospective review of 8822 singleton deliveries between January 2012 and December 2014 was conducted at four hospitals in separate Departments across Haiti. Researchers examined the proportion of women with reported HDP (hypertension, preeclampsia, eclampsia) and the association between women with HDP and three neonatal outcomes: low birth weight, preterm birth, and stillbirths; and two maternal outcomes: placental abruption and maternal death in Hôpital Albert Schweitzer (HAS). Odds ratios for associations between HDP and perinatal outcomes at HAS were assessed using logistic regression, adjusting for potential confounders.

Results: Of the 8822 singleton births included in the study, 510 (5.8\%) had a reported HDP (including 285 (55.9\%) preeclampsia, 119 (23.3\%) eclampsia, and 106 (20.8\%) hypertension). Prevalence of HDP among each hospital was: HAS (13.5\%), Hôpital Immaculée Conception des Cayes (HIC) (3.2\%), Fort Liberté (4.3\%), and Hôpital Sacré Coeur de Milot (HSC) (3.0\%). Among women at HAS with HDP, the adjusted odds of having a low birth weight baby was four times that of women without HDP (aOR 4.17, 95\% Cl 3.19-5.45), more than three times that for stillbirths (aOR 3.51, $95 \% \mathrm{Cl} 2.43-5.06$ ), and five times as likely to result in maternal death (aOR 5.13,95\% Cl 1.53-17.25). Among the three types of HDP, eclampsia was associated with the greatest odds of adverse events with five times the odds of having a low birth weight baby (aOR 5.00,95\% Cl 2.84-8.79), six times the odds for stillbirths (aOR 6.34, 95\% Cl 3.40-11.82), and more than twelve times as likely to result in maternal death (aOR 12.70, 95\% Cl 2.33-69.31).

Conclusions: A high prevalence of HDP was found among a cohort of Haitian mothers. HDP was associated with higher rates of adverse maternal and neonatal outcomes in HAS, which is comparable to studies of HDP conducted in high-income countries.
\end{abstract}

Keywords: Hypertensive disorders in pregnancy, Hypertension, Preeclampsia, Eclampsia, Pregnancy, Stillbirth, Haiti

\footnotetext{
* Correspondence: yup3@cdc.gov

${ }^{1}$ U.S. Centers for Disease Control and Prevention, Atlanta, GA, USA

Full list of author information is available at the end of the article
}

(c) The Author(s). 2019 Open Access This article is distributed under the terms of the Creative Commons Attribution 4.0 International License (http://creativecommons.org/licenses/by/4.0/), which permits unrestricted use, distribution, and reproduction in any medium, provided you give appropriate credit to the original author(s) and the source, provide a link to the Creative Commons license, and indicate if changes were made. The Creative Commons Public Domain Dedication waiver (http://creativecommons.org/publicdomain/zero/1.0/) applies to the data made available in this article, unless otherwise stated. 


\section{Background}

Haiti is known to have one of the highest maternal mortality ratios (MMR) in the world at an estimated 359 per 100,000 live births [1]. The stillbirth rate is also high with an annual estimated rate of 15.5 stillbirths per 1000 live births, resulting in 4300 stillbirths per year [2]. Hypertensive disorders in pregnancy (HDP) are one of the leading causes of maternal mortality and stillbirths [3]. HDP includes gestational hypertension, chronic hypertension, preeclampsia, and eclampsia. Hypertension (both chronic and during pregnancy) has been identified as a significant cause of morbidity among Haitian women, yet the etiology behind this association is currently unknown $[4,5,6,7]$. Few studies have attempted to explore hypertensive disorders among pregnant women in Haiti; thus, the true impact of HDP on Haitian mothers and babies is largely unknown. Low and middle-income countries (LMIC) are known to be disproportionally affected by HDP, yet good quality information on prevalence of HDP in these settings are still missing in the literature.

In 2012, the United States Centers for Disease Control and Prevention (CDC) partnered with the Haitian Ministry of Public Health and Population (MSPP) to set up an Enhanced Routine Pregnancy Outcome Surveillance System (ePOSS) in Haiti. The goal of this surveillance system is to improve the collection and analysis of routine epidemiologic indicators used to measure pregnancy outcomes at the facility and community levels in selected regions of Haiti [8]. ePOSS was initially established in three departments, Artibonite, Nord, and Nord-Est, which serve 11 communes collectively. The facilities that were targeted in these communes were tertiary healthcare facilities and/or comprehensive emergency obstetric care facilities (CEmOC).

As of October 2014, the ePOSS system had been introduced and implemented in 19 target facilities within six administrative Departments: Artibonite, Nord, Nord-Est, Sud, Sud-Est, and Nippes. Four hospitals, each representing a different department, were included in this study: Hôpital Albert Schweitzer (HAS) in Artibonite, Hôpital de Fort Liberté in Nord-Est, Hôpital Sacré Coeur de Milot (HSC) in Nord, and Hôpital Immaculée Conception des Cayes (HIC) in Sud (Fig. 1). No data were available for hospitals in Sud Est and Nippes at the time of evaluation.

Prior studies in Haiti have found a prevalence of preeclampsia and/or eclampsia among pregnant women to be $7-18 \%[9,10,11]$. One of these studies by Raghuraman et al. examined associations between HDP and adverse maternal and fetal outcomes in HAS hospital records from January 2011-December 2012 [9]. Investigators found the prevalence of preeclampsia and eclampsia to be $16.6 \%$ and an association between preeclampsia and eclampsia with maternal death and placental abruption [9]. The study was limited to data from one hospital in Haiti and was unable to compare preeclamptic and eclamptic women with normotensive women due to a lack of data collected on women who were normotensive [9].

This ongoing study will expand upon the research by Raghuraman et al. by using more recent data from four

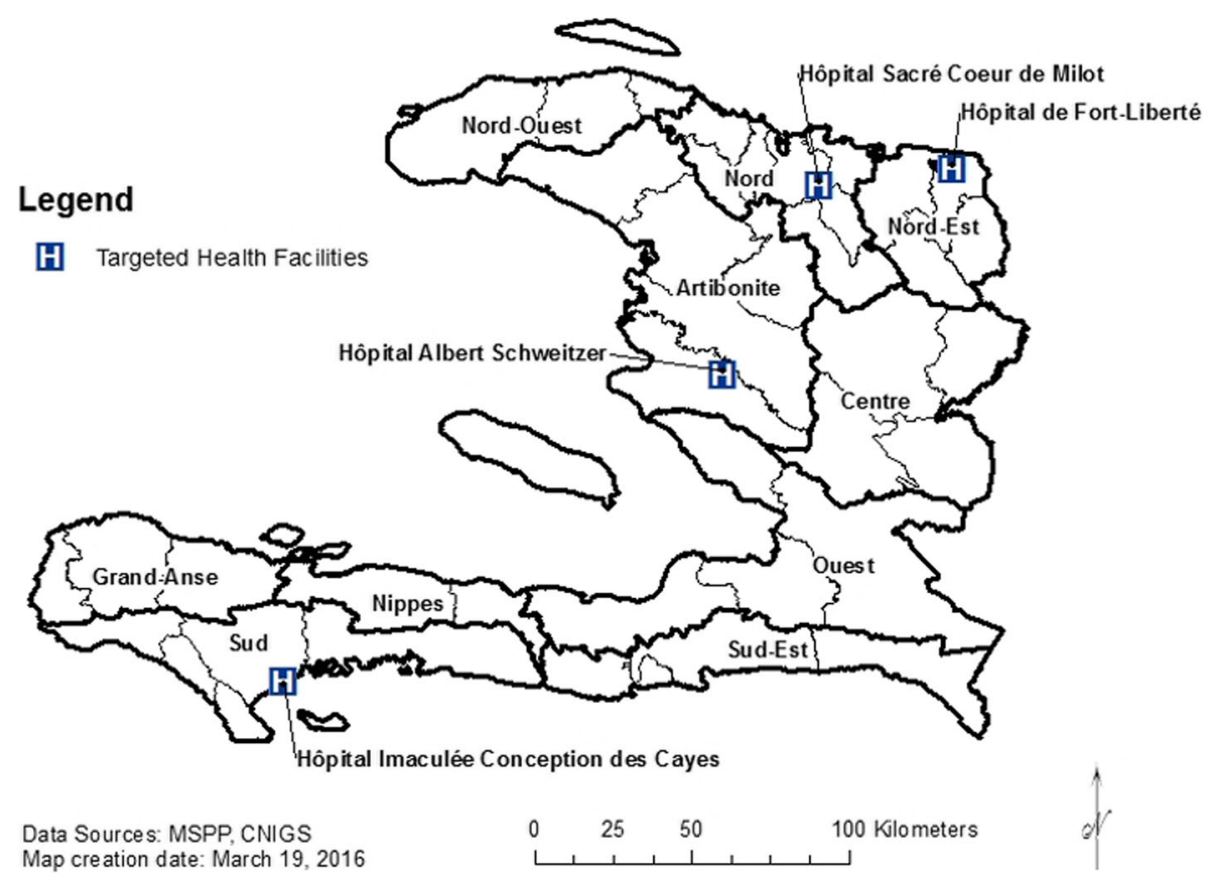

Fig. 1 Geographic Distribution of Targeted Hospitals (ePOSS, 2012-2014) 
hospitals in Haiti, including HAS, and exploring differences in adverse maternal and neonatal outcomes between hypertensive and normotensive women delivering at HAS. The aims of this study are to determine: (1) the prevalence of hypertensive disorders in pregnant women with singleton births delivering at four Departmental hospitals in Haiti; and (2) the extent to which certain maternal and neonatal outcomes are associated with these disorders. Answers to these questions add to the limited research on HDP in low resource areas by studying linkages of maternal and newborn outcomes. This study also allows researchers to identify areas for further improvement at the hospital level and provide data that can aid in evidence-based intervention strategies.

\section{Methods}

Data

Data were retrieved from the Haiti ePOSS system, which combines data from medical registries and charts. For this study, data from four hospitals (HAS, Fort Liberté, HSC, and HIC) were selected based on the following criteria: 1) being the largest referral hospitals in their distinct Departments, and 2) having the most complete records known by the research team, who participate in ePOSS. HAS and Fort Liberté had complete data from 2013 and 2014. For HSC, only 2012 data were available at the time of the analysis. For HIC, data from 2014 (its first year in ePOSS) were included.

These four hospitals are located in different Departments throughout Haiti (Fig. 1). HAS is located in Verrettes commune in Artibonite. Fort Liberté is located in the Fort Liberte commune in Nord Est; HSC is in the Milot commune in Nord; and HIC is in the Port-de-paix commune in Sud (Fig. 1).

All hospitals recorded data into multiple reporting system registries, including: maternity, major and minor operating theatre, emergency unit, and abortion (induced or late spontaneous). ePOSS is able to combine these multiple registries into a single registry for all hospitals except for HIC. Due to registry system issues, HIC still had two separate registries in ePOSS: one maternal registry and one operating theater (OT) registry. Women in the OT registry were those who required surgery, while the maternity registry captured vaginal births or low-risk cesarean sections. Both registries were used for the HDP prevalence calculation, but due to lack of data on outcomes of interest (infant status, maternal status, and birth weight) in the OT registry, only the maternal registry was used in further analyses.

The primary outcomes of interest in ePOSS are outcomes of pregnancy including maternal deaths, stillbirths, neonatal deaths, birth complications, birth defects, and live births [8]. Maternal and neonatal deaths were defined by status at discharge from hospital. An offspring's status at birth was recorded as live birth or stillbirth (macerated, fresh, or undetermined stillbirth). Maternal, neonatal, and birth complications were recorded in three columns. Up to three complications were recorded for each birth [8].

\section{Sample}

A retrospective review was conducted of 9069 women entered into ePOSS (Fig. 2). Women who had multiplebirth pregnancies $(n=237)$ or who had an induced or late spontaneous abortion $(n=10)$ were excluded from this study. There were three cohorts used in the analyses. Prevalence was estimated using the full cohort of 8822 women. Due to a lack of further data collected at HIC's OT Registry, 864 women had to be omitted from further analyses; thus, the second cohort size was 7958 women. The final cohort that analyses were run on included women from HAS only $(n=2080)$ (Fig. 2$)$.

\section{Exposure variable}

The exposure of interest was HDP, defined as a diagnosis of one the following complications during pregnancy: hypertension during pregnancy (systolic blood pressure (SBP) level $\geq 140 \mathrm{mmHg}$ or diastolic blood pressure (DBP) $\geq 90 \mathrm{mmHg}$ on at least two occasions, four or more hours apart after 20 weeks gestation), preeclampsia, and/or eclampsia. Preeclampsia was characterized as a SBP level of $\geq 140 \mathrm{mmHg}$ or $\mathrm{DBP} \geq 90 \mathrm{mmHg}$ (on at least two occasions, four or more hours apart) with proteinuria $(\geq 300 \mathrm{mg} / 24 \mathrm{~h})$ in at least 2 urine specimens collected 6 hours or more apart. Eclampsia was defined as a SBP level of $\geq 140 \mathrm{mmHg}$ or $\mathrm{DBP} \geq 90 \mathrm{mmHg}$, proteinuria, and seizures $[12,13]$.

\section{Outcome variables}

The variables included in this study were maternal and neonatal outcomes that are thought to be associated with HDP based on current research in high-income countries $[14,15]$. The main neonatal outcomes of interest were low birth weight $(<2500 \mathrm{~g})$, preterm birth $(<37$ weeks' gestation), and stillbirths (death at or after 28 weeks' gestation). The two maternal outcomes of interest were placental abruption and maternal death (prior to hospital discharge). Other variables included in the analyses were maternal age, parity (dichotomized as nulliparous vs. parous), mode of delivery (vaginal vs. Caesarean section), and gestational age in weeks.

\section{Data analysis}

Comparative analyses, including prevalence, between women with HDP and those without were conducted between the hospitals. Continuous data were summarized using mean and standard deviation and categorical data were presented as frequencies and percentages. Comparisons were assessed using T-tests for continuous variables, chi-square tests for categorical variables, and 


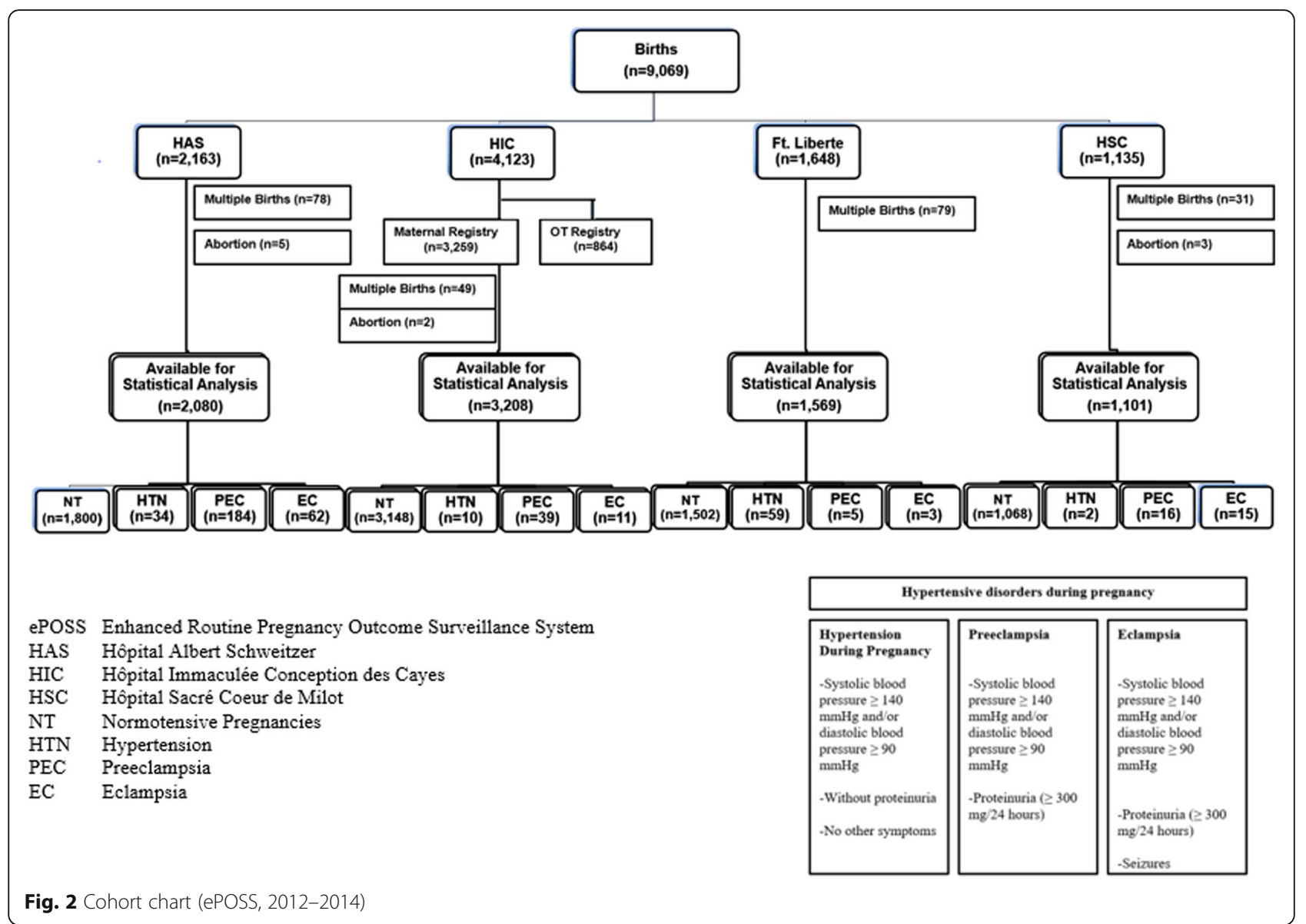

Fisher's exact tests for categorical variables with small values. All significant thresholds were set at a $p$-value $<0.05$.

Logistic regression was used to calculate adjusted odds ratios with 95\% confidence intervals using HAS data exclusively (2080 pregnancies). The outcomes analyzed were low birth weight, stillbirth, and maternal death. There were not enough cases of placental abruption to analyze further. Odds ratios were adjusted for the following potential confounders: maternal age, parity, and year of delivery (to account for time of data recording).

Statistical analyses were performed using SAS 9.4 (Cary, NC).

\section{Results}

\section{HDP prevalence}

Among the 8822 women with singleton births included in the study, $510(5.8 \%)$ had a HDP. Prevalence of HDP among the selected sample at each hospital was as follows: HAS (13.5\%), HIC (3.2\%), Fort Liberté (4.3\%) and HSC (3.0\%). Among the 510 women with HDP, 285 (55.9\%) were preeclamptic, 119 (23.3\%) were eclamptic, and 106 (20.8\%) had gestational hypertension (Table 1).

\section{Study population characteristics and obstetric and perinatal outcomes}

Of the 8822 women included in the study, clinical characteristics were available for 7958 (90.2\%). These women had a mean maternal age at delivery of 27.7 years $(\mathrm{SD}=$ 6.7 ) and 4194 (52.7\%) women had a prior delivery. Of the 7958 women included in the analysis, 6263 (78.7\%) had a vaginal delivery. There were 738 (9.3\%) preterm deliveries, 1240 (15.6\%) low birth weight babies, and 392 (4.9\%) stillbirths. The stillbirth rate for this cohort was 49.3 stillbirths per 1000 live births. There were $26(0.3 \%)$ maternal deaths and $38(0.5 \%)$ women who had a placental abruption (Table 2). The MMR in this study, classified as deaths prior to discharge, was found to be 327 deaths per 100,000 live births.

\section{Characteristics among HDP vs. no HDP associated with outcome variables}

Table 2 also shows characteristics and outcomes by hospital and by HDP status. In all hospitals except Fort Liberté, women with HDP were significantly more likely to have a low birth weight baby $(p<0.05)$. Women at HAS and HIC with HDP had significantly higher proportions of preterm deliveries and stillbirths. Maternal death was 
Table 1 Prevalence of hypertensive disorders in pregnancy in 4 hospitals by 6 month intervals (ePOSS, 2012-2014)

\begin{tabular}{|c|c|c|c|c|c|c|c|c|}
\hline & & Jan-June 2012 & July-Dec 2012 & Jan-June 2013 & July-Dec 2013 & Jan-June 2014 & July-Dec 2014 & Overall Hospital Total \\
\hline & & $\begin{array}{c}\text { Number of HDP } \\
\text { Cases } \\
\text { (Hospital Specific } \\
\text { Prevalence) }\end{array}$ & $\begin{array}{c}\text { Number of HDP } \\
\text { Cases } \\
\text { (Hospital Specific } \\
\text { Prevalence) }\end{array}$ & $\begin{array}{c}\text { Number of HDP } \\
\text { Cases } \\
\text { (Hospital Specific } \\
\text { Prevalence) }\end{array}$ & $\begin{array}{c}\text { Number of HDP } \\
\text { Cases } \\
\text { (Hospital Specific } \\
\text { Prevalence) }\end{array}$ & $\begin{array}{c}\text { Number of HDP } \\
\text { Cases } \\
\text { (Hospital Specific } \\
\text { Prevalence) }\end{array}$ & $\begin{array}{c}\text { Number of HDP } \\
\text { Cases } \\
\text { (Hospital Specific } \\
\text { Prevalence) }\end{array}$ & $\begin{array}{c}\text { Total Number of HDP } \\
\text { Cases } \\
\text { (Total Hospital Specific } \\
\text { Prevalence) }\end{array}$ \\
\hline \multirow{4}{*}{$\begin{array}{c}\text { HAS } \\
(n=2080)\end{array}$} & Hypertension & & & $1(0.2)$ & $16(2.8)$ & $10(2.0)$ & $7(1.3)$ & $34(1.6)$ \\
\hline & Preeclampsia & & & $0(0.0)$ & $78(13.8)$ & $54(11.1)$ & $52(9.7)$ & $184(8.8)$ \\
\hline & Eclampsia & & & $0(0.0)$ & $34(6.0)$ & $10(2.0)$ & $18(3.3)$ & $62(3.0)$ \\
\hline & Total HDP in HAS & -- & --- & $1(0.2)$ & $128(22.6)$ & $74(15.1)$ & $77(14.3)$ & $280(13.5)$ \\
\hline \multirow{4}{*}{$\underset{(n=4072)}{\text { HIC }}$} & Hypertension & & & & & $0(0.0)$ & $11(0.5)$ & $11(0.3)$ \\
\hline & Preeclampsia & & & & & $15(0.8)$ & $65(2.9)$ & $80(2.0)$ \\
\hline & Eclampsia & & & & & $12(0.7)$ & $27(1.2)$ & $39(1.0)$ \\
\hline & Total HDP in HIC & $\ldots$ & --- & --- & --- & $27(1.5)$ & $103(4.6)$ & $130(3.2)$ \\
\hline \multirow{4}{*}{$\begin{array}{c}\text { Fort Liberté } \\
(\mathrm{n}=1569)\end{array}$} & Hypertension & & & $4(1.0)$ & $14(2.8)$ & 33 (11.6) & $8(2.1)$ & $59(3.8)$ \\
\hline & Preeclampsia & & & $0(0.0)$ & $0(0.0)$ & $0(0.0)$ & $5(1.3)$ & $5(0.3)$ \\
\hline & Eclampsia & & & $2(0.5)$ & $0(0.0)$ & $1(0.4)$ & $0(0.0)$ & $3(0.2)$ \\
\hline & $\begin{array}{l}\text { Total HDP in Fort } \\
\text { Liberté }\end{array}$ & --- & --- & $6(1.5)$ & $14(2.8)$ & 34 (11.9) & $13(3.4)$ & $67(4.3)$ \\
\hline \multirow{4}{*}{$\begin{array}{c}\text { HSC } \\
(n=1101)\end{array}$} & Hypertension & $0(0.0)$ & $2(0.3)$ & & & & & $2(0.2)$ \\
\hline & Preeclampsia & $10(1.9)$ & $6(1.0)$ & & & & & $16(1.4)$ \\
\hline & Eclampsia & $9(1.7)$ & $6(1.0)$ & & & & & $15(1.4)$ \\
\hline & Total HDP in HSC & $19(3.7)$ & $14(2.4)$ & --- & --- & --- & --- & $33(3.0)$ \\
\hline \multirow{4}{*}{$\underset{\substack{\text { Total } \\
(\mathbf{n}=\mathbf{8 8 2 2})}}{ }$} & Hypertension & & & & & & & $106(1.2)$ \\
\hline & Preeclampsia & & & & & & & $285(3.2)$ \\
\hline & Eclampsia & & & & & & & $119(1.3)$ \\
\hline & Total HDP & & & & & & & $510(5.8)$ \\
\hline
\end{tabular}

significantly linked to HDP status at HAS only $(p=$ 0.002), Placenta abruption was significantly more likely among women with HDP in comparison to those without HDP in all hospitals except Fort Liberté $(\mathrm{p}<0.05)$.

\section{Characteristics among stillbirths vs. live births at HAS}

There were 156 (7.5\%) stillbirths and 1917 (92.2\%) live births at HAS; outcome data were not available for seven $(0.3 \%)$ births (Table 3). Women with stillbirths were more likely to be parous and more likely to have a vaginal birth. A stillbirth was more likely to be low birth weight. A live birth was more common among normotensive mothers, while stillbirths were more common among mothers with preeclampsia or eclampsia. A stillbirth was also significantly associated with maternal death and placenta abruptions.

Among the 2080 women giving birth at HAS, 280 presented with HDP. Among these women with HDP, there were 52 (18.6\%) stillbirths and 227 (81.1\%) live births; mortality information was not available for one woman. There was no significant difference in maternal age by infant outcome. Among women with HDP at HAS, stillbirths were more likely than live births to be parous and have a vaginal delivery. More placental abruptions were seen among the women having stillbirths (Table 3).

\section{Characteristics among maternal deaths vs. survivors at HAS}

As seen in Table 4, there were 11 maternal deaths $(0.5 \%$ of women with singleton deliveries) at HAS; maternal status was not available for nine women. Women who died at HAS were more likely to have had a Caesarean section and deliver a low birth weight baby. Women who died were more likely to suffer from eclampsia and less likely to be normotensive. Those with a maternal death were also significantly more likely to deliver a stillborn baby.

Of the 280 women at HAS with HDP, 274 mothers (97.9\%) survived pregnancy and five mothers died; vital status was not available for one mother. Women with HDP who died were older (36.0 years vs. 28.3 years) and more likely to have a Caesarean section delivery. A larger proportion of these women were parous, but the difference was not statistically significant. All other adverse outcomes were not significantly different between the two groups (Table 4).

\section{Association between HDP and adverse outcomes}

Among women at HAS who had HDP, the adjusted odds of having a low birth weight baby was four times that for women without HDP (aOR 4.17, 95\% CI 3.19-5.45), more than three times for stillbirths (aOR 3.51, 95\% CI 2.43-5.06), and five times for maternal death (aOR 5.13, 95\% CI 1.53-17.25). Among the 3 types of HDP, eclampsia was associated with the greatest odds of adverse events with five times the odds of having a low birth weight baby (aOR 5.00, 95\% CI 2.84-8.79), six times for stillbirths (aOR 6.34, 95\% CI 3.40-11.82), and more than twelve times for maternal death (aOR 12.70, 95\% CI 2.33-69.31). Hypertension alone was not associated with adverse outcomes. Crude odds ratios were similar to the adjusted odds ratios (Table 5). 
Table 2 Comparison of HDP vs. No HDP and obstetric characteristics of women at 4 hospitals in Haiti (ePOSS, 2012-2014)

\begin{tabular}{|c|c|c|c|c|c|c|c|c|c|c|c|c|c|}
\hline & $\begin{array}{l}\text { Full Cohort } \\
(\mathrm{n}=7958)\end{array}$ & $\begin{array}{c}\text { HAS } \\
\text { No HDP } \\
(n=1800)\end{array}$ & $\begin{array}{c}\text { HAS } \\
\text { HDP } \\
(\mathrm{n}=280)\end{array}$ & $\mathbf{p}^{\mathrm{a}}$ & $\begin{array}{c}\text { HIC } \\
\text { No HDP } \\
(\mathbf{n}=3148)^{*}\end{array}$ & $\begin{array}{c}\text { HIC } \\
\text { HDP } \\
(\mathbf{n}=60)^{*}\end{array}$ & $\mathbf{p}^{\mathrm{b}}$ & $\begin{array}{c}\text { Fort Liberté } \\
\text { No HDP } \\
(\mathrm{n}=1502)\end{array}$ & $\begin{array}{c}\text { Fort Liberté } \\
\text { HDP } \\
(n=67)\end{array}$ & $\mathbf{p}^{\mathrm{c}}$ & $\begin{array}{c}\text { HSC } \\
\text { No HDP } \\
(n=1068)\end{array}$ & $\begin{array}{c}\text { HSC } \\
\text { HDP } \\
(n=33)\end{array}$ & $\mathbf{p}^{\mathrm{d}}$ \\
\hline$\overline{\text { Maternal age (years) }}{ }^{e}$ & $27.7 \pm 6.7$ & $27.6 \pm 6.8$ & $28.4 \pm 8.0$ & 0.08 & $27.7 \pm 6.5$ & $28.4 \pm 8.0$ & 0.46 & $26.8 \pm 6.4$ & $28.8 \pm 6.8$ & 0.01 & $29.1 \pm 6.4$ & $26.8 \pm 7.4$ & 0.05 \\
\hline Parity & & & & 0.02 & & & 0.61 & & & 0.58 & & & 0.44 \\
\hline Nulliparous & $3483(43.8)$ & $836(46.4)$ & $109(38.9)$ & & $1332(42.3)$ & $23(38.3)$ & & $617(41.1)$ & $30(44.8)$ & & $523(49.0)$ & $13(39.4)$ & \\
\hline Parous & $4194(52.7)$ & $962(53.4)$ & $171(61.1)$ & & $1556(49.4)$ & $31(51.7)$ & & $874(58.2)$ & $37(55.2)$ & & $545(51.0)$ & $18(54.5)$ & \\
\hline Missing & $281(3.5)$ & $2(0.1)$ & $0(0.0)$ & & $260(8.3)$ & $6(10.0)$ & & $11(0.7)$ & $0(0.0)$ & & $0(0.0)$ & $2(6.1)$ & \\
\hline Vaginal & $6263(78.7)$ & $1259(69.9)$ & $179(63.9)$ & & $2496(79.3)$ & $38(63.3)$ & & 1421 (94.6) & $61(91.0)$ & & $808(75.7)$ & $1(3.0)$ & \\
\hline Caesarean section & $1310(16.5)$ & $541(30.1)$ & $101(36.1)$ & & $290(9.2)$ & $10(16.7)$ & & $76(5.1)$ & $6(9.0)$ & & $254(23.8)$ & $32(97.0)$ & \\
\hline Missing & $385(4.8)$ & $0(0.0)$ & $0(0.0)$ & & $362(11.5)$ & $12(20.0)$ & & $5(0.3)$ & $0(0.0)$ & & $6(0.6)$ & $0(0.0)$ & \\
\hline Low Birthweight $(<2500 \mathrm{~g})$ & & & & $<0.0001$ & & & 0.0004 & & & 0.80 & & & 0.0006 \\
\hline Yes & $1240(15.6)$ & $394(21.9)$ & $149(53.2)$ & & $343(10.9)$ & $14(23.3)$ & & $135(9.0)$ & $7(10.5)$ & & $186(17.4)$ & $12(36.4)$ & \\
\hline No & $5667(71.2)$ & $1327(73.7)$ & $121(43.2)$ & & $2409(76.5)$ & $33(55.0)$ & & $900(59.9)$ & $42(62.7)$ & & $823(77.1)$ & $12(36.4)$ & \\
\hline Missing & $1051(13.2)$ & $79(4.4)$ & $10(3.6)$ & & $396(12.6)$ & $13(21.7)$ & & $467(31.1)$ & $18(26.9)$ & & $59(5.5)$ & $9(27.3)$ & \\
\hline Preterm delivery ( $<37$ weeks) & & & & $<0.0001$ & & & $<0.0001$ & & & 0.30 & & & --- \\
\hline Yes & $738(9.3)$ & $179(9.9)$ & $78(27.9)$ & & $241(7.7)$ & $13(21.7)$ & & $211(14.0)$ & $16(23.9)$ & & --- & --- & \\
\hline No & 3880 (48.8) & $991(55.1)$ & $135(48.2)$ & & $2163(68.7)$ & $30(50.0)$ & & $532(35.4)$ & $29(43.3)$ & & --- & --- & \\
\hline Missing & $3340(42.0)$ & $630(35.0)^{\mathrm{g}}$ & $67(23.9)^{\mathrm{g}}$ & & $744(23.6)$ & $17(28.3)$ & & $759(50.5)$ & $22(32.8)$ & & $1068(100.0)^{g}$ & $33(100.0)^{\mathrm{g}}$ & \\
\hline Stillbirth & & & & $<0.0001$ & & & 0.01 & & & 0.15 & & & 0.08 \\
\hline Yes & $392(4.9)$ & $104(5.8)$ & $52(18.6)$ & & $124(3.9)$ & $6(10.0)$ & & $46(3.1)$ & $0(0.0)$ & & $56(5.2)$ & $4(12.1)$ & \\
\hline No & $7168(90.1)$ & $1690(93.9)$ & $227(81.1)$ & & $2652(84.2)$ & $40(66.7)$ & & 1454 (96.8) & $67(100.0)$ & & $1010(94.6)$ & $28(84.9)$ & \\
\hline Missing & $398(5.0)$ & $6(0.3)$ & $1(0.4)$ & & $372(11.8)$ & $14(23.3)$ & & $2(0.1)$ & $0(0.0)$ & & $2(0.2)$ & $1(3.0)$ & \\
\hline Maternal death & & & & 0.002 & & & 0.98 & & & 0.55 & & & 0.68 \\
\hline Yes & $26(0.3)$ & $6(0.3)$ & $5(1.8)$ & & $1(0.03)$ & $0(0.0)$ & & $8(0.5)$ & $0(0.0)$ & & $6(0.5)$ & $0(0.0)$ & \\
\hline No & $6043(75.9)$ & $1786(99.2)$ & $274(97.9)$ & & $1370(43.5)$ & $1(1.7)$ & & 1455 (96.9) & $66(98.5)$ & & $1061(99.3)$ & $30(90.9)$ & \\
\hline Missing & 1889 (23.7) & $8(0.4)$ & $1(0.4)$ & & $1777(56.4)$ & $59(98.3)$ & & $39(2.6)$ & $1(1.5)$ & & $1(0.1)$ & $3(9.1)$ & \\
\hline Placenta abruption & & & & 0.04 & & & $<0.0001$ & & & 0.83 & & & $<0.0001$ \\
\hline Yes & $38(0.5)$ & $11(0.6)$ & $5(1.8)$ & & $7(0.2)$ & $2(3.3)$ & & $1(0.1)$ & $0(0.0)$ & & $8(0.7)$ & $4(12.1)$ & \\
\hline No & 7920 (99.5) & 1789 (99.4) & $275(98.2)$ & & 3141 (99.8) & $58(96.7)$ & & $1501(99.9)$ & $67(100.0)$ & & $1060(99.3)$ & $29(87.9)$ & \\
\hline \multicolumn{14}{|c|}{ Data are shown as mean $\pm \mathrm{SD}$ or $\mathrm{n}(\%)$. } \\
\hline \multicolumn{14}{|c|}{ * HIC data from Maternal Registry Only } \\
\hline \multicolumn{14}{|c|}{ 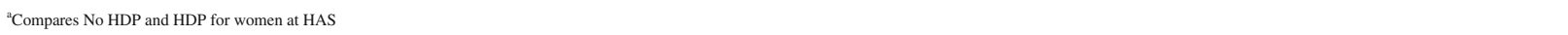 } \\
\hline \multicolumn{14}{|c|}{${ }^{\mathrm{b}}$ Compares No HDP and HDP for women at HIC } \\
\hline \multicolumn{14}{|c|}{${ }^{\circ}$ Compares No HDP and HDP for women at Fort Liberte } \\
\hline \multicolumn{14}{|c|}{${ }^{\mathrm{d} C}$ Compares No HDP and HDP for women at Milot } \\
\hline \multicolumn{14}{|c|}{${ }^{\mathrm{e}} 411$ mothers missing maternal age $(5.2 \%)$} \\
\hline$f_{3,340 \text { mothers missing gestational }}$ & age $(42.0 \%)$ & & & & & & & & & & & & \\
\hline${ }^{g}$ Gestational age was not collecte & until July 20 & it is mi & & & & & & & & & & & \\
\hline
\end{tabular}

\section{Discussion}

This study examines the prevalence of HDP among Haitians, while also investigating the link between HDP and adverse pregnancy outcomes. Women with HDP in this sample were more likely to have pregnancies that resulted in low birth weight babies, stillbirths, and maternal death. Women with eclampsia were at greatest risk for these adverse maternal and neonatal outcomes.

It is estimated that HDP occur in 5-10\% of all pregnancies worldwide [16]. Low and middle-income countries are known to be disproportionally affected by HDP. The few prior studies in Haiti have estimated prevalence of preeclampsia and/or eclampsia among their samples to be 7$18 \%[9,10,11]$. This hospital-based study found an overall HDP prevalence to be $5.8 \%$, with individual hospitals' prevalence ranging from 3.0 to $13.5 \%$. Three of the facilities (HIC, Fort Liberté, and HSC) have similar estimates, while HAS has much higher prevalence. While all 4 facilities are referral hospitals, HAS is known to be the largest referral hospital with the most established electronic medical record (EMR) system and sees the most complicated cases. It is difficult to tell whether differences in prevalence rates are due to EMR reporting and what is due to occurrence of disease in the populations presenting at the various facilities.

Haiti's MMR is estimated to be between 359 and 389 deaths per 100,000 live births [1]. Although this study classified maternal mortality based on deaths occurring prior to discharge, a similar MMR of 327 deaths per 100,000 live births was found. The research team expects more maternal deaths would be captured if these mothers were followed for 42 days post-delivery. The lack of postpartum data is a known limitation, as a significant proportion of maternal deaths occur after women leave the hospital. Additionally, many women with complications never reach facilities, which may 
Table 3 Clinical presentation of women with and without stillbirths at HAS, by entire cohort and by HDP mothers only (ePOSS, 2012-2014)

\begin{tabular}{|c|c|c|c|c|c|c|c|}
\hline \multicolumn{5}{|c|}{ Entire HAS Cohort (n=2080) } & \multicolumn{3}{|c|}{ HDP women only $(n=280)^{b}$} \\
\hline & $\begin{array}{l}\text { Live birth } \\
(n=1917)\end{array}$ & $\begin{array}{c}\text { Stillbirth } \\
(n=156)\end{array}$ & $\mathbf{p}$ & $\begin{array}{c}\text { Unknown } \\
(\mathbf{n}=7)\end{array}$ & $\begin{array}{l}\text { Live birth } \\
(\mathrm{n}=\mathbf{2 2 7})\end{array}$ & $\begin{array}{l}\text { Stillbirth } \\
(\mathbf{n}=52)\end{array}$ & $\mathbf{p}$ \\
\hline Maternal age (years) ${ }^{\mathrm{e}}$ & $27.6 \pm 6.9$ & $28.9 \pm 7.8$ & 0.08 & $31.6 \pm 7.0$ & $28.1 \pm 7.9$ & $29.5 \pm 8.3$ & 0.27 \\
\hline Parity & & & $0.001 *$ & & & & $0.047 *$ \\
\hline Nulliparous & $892(46.5)$ & $52(33.3)$ & & $1(14.3)$ & 95 (41.9) & $14(26.9)$ & \\
\hline Parous & $1023(53.4)$ & $104(66.7)$ & & $6(85.7)$ & $132(58.2)$ & $38(73.1)$ & \\
\hline Missing & $2(0.1)$ & $0(0.0)$ & & $0(0.0)$ & $0(0.0)$ & $0(0.0)$ & \\
\hline Mode of Delivery & & & $0.006 *$ & & & & $0.0005 *$ \\
\hline Vaginal & $1309(68.3)$ & $123(78.8)$ & & $6(85.7)$ & $134(59.0)$ & $44(84.6)$ & \\
\hline Caesarean section & $608(31.7)$ & $33(21.2)$ & & $1(14.3)$ & $93(41.0)$ & $8(15.4)$ & \\
\hline Missing & $0(0.0)$ & $0(0.0)$ & & $0(0.0)$ & $0(0.0)$ & $0(0.0)$ & \\
\hline Low Birthweight (<2500 g) & & & $<0.0001 *$ & & & & $<0.0001 *$ \\
\hline Yes & 435 (22.7) & $108(69.2)$ & & $0(0.0)$ & $107(47.1)$ & $42(80.8)$ & \\
\hline No & $1404(73.2)$ & $43(27.6)$ & & $1(14.3)$ & $114(50.2)$ & $7(13.5)$ & \\
\hline Missing & $78(4.07)$ & $5(3.2)$ & & $6(85.7)$ & $6(2.6)$ & $3(5.8)$ & \\
\hline Gestational age (weeks) $^{\mathrm{a}}$ & $38.7 \pm 3.1$ & $32.9 \pm 6.4$ & $<0.0001$ & $35.0 \pm 5.7$ & $37.0 \pm 3.9$ & $32.4 \pm 5.3$ & $<0.0001$ \\
\hline Preterm delivery (<37 weeks) & & & $<0.0001^{*}$ & & & & $0.05 *$ \\
\hline Yes & $192(10.0)$ & $64(41.0)$ & & $1(14.3)$ & $58(25.6)$ & $20(38.5)$ & \\
\hline No & $1088(56.8)$ & $37(23.7)$ & & $1(14.3)$ & $126(55.5)$ & $9(17.3)$ & \\
\hline Missing $^{\mathrm{a}}$ & $637(33.2)$ & $55(35.3)$ & & $5(71.4)$ & $43(18.9)$ & $23(44.2)$ & \\
\hline \multicolumn{8}{|l|}{ Diagnosis } \\
\hline Normotensive & $1690(88.2)$ & $104(66.7)$ & $<0.0001 *$ & $6(85.7)$ & -- & -- & -- \\
\hline Hypertension & $32(1.7)$ & $2(1.3)$ & 0.74 & $0(0.0)$ & $32(14.1)$ & $2(3.9)$ & $0.04 *$ \\
\hline Preeclampsia & $150(7.8)$ & $34(21.8)$ & $<0.0001 *$ & $0(0.0)$ & $150(66.1)$ & $34(65.4)$ & $0.92 *$ \\
\hline Eclampsia & $45(2.3)$ & $16(10.3)$ & $<0.0001$ & $1(14.3)$ & $45(19.8)$ & $16(30.77)$ & 0.09 \\
\hline Maternal death & & & 0.007 & & & & 0.23 \\
\hline Yes & $7(0.4)$ & $4(2.6)$ & & $0(0.0)$ & $3(1.3)$ & $2(3.85)$ & \\
\hline No & $1902(99.2)$ & $151(96.8)$ & & $7(100.0)$ & $224(98.7)$ & $49(94.2)$ & \\
\hline Missing & $8(0.4)$ & $1(0.6)$ & & $0(0.0)$ & $0(0.0)$ & $1(1.9)$ & \\
\hline Placenta abruption & & & $<0.0001^{*}$ & & & & 0.01 \\
\hline Yes & $7(0.4)$ & $9(5.8)$ & & $0(0.0)$ & $1(0.4)$ & $4(7.7)$ & \\
\hline No & $1910(99.6)$ & $147(94.2)$ & & $7(100.0)$ & $226(99.6)$ & $48(92.3)$ & \\
\hline Missing & $0(0.0)$ & $0(0.0)$ & & $0(0.0)$ & $0(0.0)$ & $0(0.0)$ & \\
\hline \multicolumn{8}{|c|}{ ePOSS, Enhanced Routine Pregnancy Surveillance System; HDP, Hypertensive disorder in pregnancy; HAS, Hôpital Albert Schweitzer } \\
\hline \multicolumn{8}{|c|}{ Data are shown as mean $\pm \mathrm{SD}$ or $\mathrm{n}(\%)$} \\
\hline \multicolumn{8}{|c|}{ Statistically significant P-values are shown in bold. } \\
\hline \multicolumn{8}{|c|}{ *P-value calculated using chi-square, all other P-values calculated by Fisher's exact test. } \\
\hline \multicolumn{8}{|c|}{ a'Gestational age was not collected until July 2013; thus, it is missing if prior } \\
\hline${ }^{\mathrm{b}}$ Missing 1 observation for Stillbirth sta & & & & & & & \\
\hline
\end{tabular}

also lead to an underreporting of maternal mortality, HDP and other adverse outcomes.

The estimated stillbirth rate for Haiti is 15.5 stillbirths per 1000 live births [2]. The current study found a much higher stillbirth rate of 49.3 stillbirths per 1000 live births at HAS. It is likely that this hospital-based sample does not represent the true prevalence of major complications (HDP, maternal death, and stillbirth). Women without complications may be less likely to deliver at a facility, which could result in biased estimates as well. 
Table 4 Clinical presentation of women by maternal death status at HAS, by entire cohort and by HDP women only (ePOSS, 20122014)

\begin{tabular}{|c|c|c|c|c|c|c|c|}
\hline \multicolumn{5}{|c|}{ Entire HAS Cohort $(n=2080)$} & \multicolumn{3}{|c|}{ HDP women only $(n=280)^{b}$} \\
\hline & $\begin{array}{c}\text { No maternal } \\
\text { death }(n=2060)\end{array}$ & $\begin{array}{c}\text { Maternal } \\
\text { death }(n=11)\end{array}$ & $\mathbf{p}$ & $\begin{array}{c}\text { Unknown } \\
(\mathbf{n}=9)\end{array}$ & $\begin{array}{c}\text { No maternal } \\
\text { death }(n=274)\end{array}$ & $\begin{array}{c}\text { Maternal } \\
\text { death }(n=5)\end{array}$ & $\mathbf{p}$ \\
\hline Maternal age $(\text { years })^{\mathrm{e}}$ & $27.7 \pm 7.0$ & $30.9 \pm 8.4$ & 0.13 & $28.3 \pm 8.6$ & $28.3 \pm 7.9$ & $36.0 \pm 7.0$ & $\mathbf{0 . 0 3}$ \\
\hline Parity & & & $0.07 *$ & & & & 0.08 \\
\hline Nulliparous & $940(45.6)$ & $2(18.1)$ & & $3(33.3)$ & $109(39.8)$ & $0(0.0)$ & \\
\hline Parous & $1118(54.3)$ & $9(81.8)$ & & $6(66.7)$ & $165(60.2)$ & $5(100.0)$ & \\
\hline Missing & $2(0.001)$ & $0(0.0)$ & & $0(0.0)$ & $0(0.0)$ & $0(0.0)$ & \\
\hline Mode of Delivery & & & 0.001 & & & & $\mathbf{0 . 0 1}$ \\
\hline Vaginal & $1430(69.4)$ & $2(18.1)$ & & $6(66.7)$ & $178(65.0)$ & $0(0.0)$ & \\
\hline Caesarean section & $630(30.6)$ & $9(81.8)$ & & $3(33.3)$ & $96(35.0)$ & $5(100.0)$ & \\
\hline Missing & $0(0.0)$ & $0(0.0)$ & & $0(0.0)$ & $0(0.0)$ & $0(0.0)$ & \\
\hline Low Birthweight $(<2500 \mathrm{~g})$ & & & 0.01 & & & & 0.26 \\
\hline Yes & $535(26.0)$ & $7(63.6)$ & & $1(11.1)$ & 145 (52.9) & $4(80.0)$ & \\
\hline No & $1437(69.8)$ & $4(36.4)$ & & $7(77.8)$ & $120(43.8)$ & $1(20.0)$ & \\
\hline Missing & $88(4.3)$ & $0(0.0)$ & & $1(11.1)$ & $9(3.3)$ & $0(0.0)$ & \\
\hline Gestational age (weeks) $^{\mathrm{a}}$ & $38.3 \pm 3.8$ & $36.1 \pm 5.0$ & 0.11 & $39.7 \pm 1.3$ & $36.4 \pm 4.3$ & $34.6 \pm 5.8$ & 0.37 \\
\hline Preterm delivery (<37 weeks) & & & 0.17 & & & & 0.60 \\
\hline Yes & $254(12.3)$ & $3(27.3)$ & & $0(0.0)$ & $76(27.7)$ & $2(40.0)$ & \\
\hline No & $1114(54.1)$ & $5(45.5)$ & & $7(77.8)$ & $132(48.2)$ & $3(60.0)$ & \\
\hline Missing $^{\mathrm{a}}$ & $692(33.6)$ & $3(27.3)$ & & $2(22.2)$ & $66(24.1)$ & $0(0.0)$ & \\
\hline \multicolumn{8}{|l|}{ Diagnosis } \\
\hline Normotensive & $1786(86.7)$ & $6(54.5)$ & 0.01 & $8(88.9)$ & -- & -- & -- \\
\hline Hypertension & $34(1.7)$ & $0(0.0)$ & 1.00 & $0(0.0)$ & $34(12.4)$ & $0(0.0)$ & 1.00 \\
\hline Preeclampsia & $181(8.8)$ & $3(27.3)$ & 0.07 & $0(0.0)$ & $181(66.1)$ & $3(60.0)$ & 0.78 \\
\hline Eclampsia & $59(2.9)$ & $2(18.2)$ & 0.04 & $1(11.1)$ & $59(21.5)$ & $2(40.0)$ & 0.30 \\
\hline Stillbirth & & & 0.01 & & & & 0.23 \\
\hline Yes & $151(7.3)$ & $4(36.4)$ & & $1(11.1)$ & $49(17.9)$ & $2(40.0)$ & \\
\hline No & $1902(92.3)$ & $7(63.6)$ & & $8(88.9)$ & $224(81.8)$ & $3(60.0)$ & \\
\hline Missing & $7(0.3)$ & $0(0.0)$ & & $0(0.0)$ & $1(0.4)$ & $0(0.0)$ & \\
\hline Placenta abruption & & & 0.08 & & & & 0.09 \\
\hline Yes & $15(0.7)$ & $1(9.1)$ & & $0(0.0)$ & $4(1.5)$ & $1(20.0)$ & \\
\hline No & $2045(99.3)$ & $10(90.9)$ & & $9(100.0)$ & $270(98.5)$ & $4(80.0)$ & \\
\hline Missing & $0(0.0)$ & $0(0.0)$ & & $0(0.0)$ & $0(0.0)$ & $0(0.0)$ & \\
\hline \multicolumn{8}{|l|}{ Data are shown as mean \pm SD or $n(\%)$} \\
\hline \multicolumn{8}{|c|}{ Statistically significant P-values are shown in bold. } \\
\hline \multicolumn{8}{|c|}{ *P-value calculated using chi-square, all other P-values calculated by Fisher's exact test. } \\
\hline \multicolumn{8}{|c|}{ a'Gestational age was not collected until July 2013; thus, it is missing if prior } \\
\hline${ }^{\mathrm{b}}$ Missing 1 observation for Maternal de & status & & & & & & \\
\hline
\end{tabular}

One of the few studies that aimed to study HDP in Haiti was by Raghuraman et al. This study, while similar in topical area, included different diagnoses than the current study; thus, the prevalence in the two studies are not comparable.
This study, similar to the current study, was also conducted at HAS. The previous study used data from 2011 to 2012, while the current study used data from 2013 to 2014. Researchers in the prior study obtained medical records from 
Table 5 Logistic regression on the association between HDP and outcomes for women at HAS (ePOSS, 2012-2014)

\begin{tabular}{|c|c|c|c|c|}
\hline \multirow[b]{2}{*}{ Outcomes } & \multicolumn{4}{|c|}{ NT vs. All HDP } \\
\hline & $\mathbf{O R}$ & $95 \% \mathrm{CI}$ & $\mathbf{a O R}^{*}$ & $95 \% \mathrm{Ci}$ \\
\hline Low Birthweight $(<2500$ g) & 4.15 & $3.18-5.41$ & 4.17 & $3.19-5.45$ \\
\hline Stillbirth & 3.72 & $2.60-5.34$ & 3.51 & 2.43-5.06 \\
\hline \multirow[t]{2}{*}{ Maternal death } & 5.43 & $1.65-17.92$ & 5.13 & 1.53-17.25 \\
\hline & \multicolumn{4}{|c|}{ NT vs. HTN } \\
\hline Outcomes & $\overline{\mathrm{OR}}$ & $95 \% \mathrm{CI}$ & $\mathbf{a O R}^{*}$ & $95 \% \mathrm{Ci}$ \\
\hline Low Birthweight $(<2500$ g) & 1.47 & $0.69-3.10$ & 1.49 & $0.70-3.18$ \\
\hline Stillbirth & 1.02 & $0.24-4.30$ & 0.87 & $0.20-3.75$ \\
\hline \multirow[t]{2}{*}{ Maternal death } & 3.98 & $0.03-34.83$ & 4.14 & $0.03-35.76$ \\
\hline & \multicolumn{4}{|c|}{ NT vs. PEC } \\
\hline Outcomes & $\mathbf{O R}$ & $95 \% \mathrm{CI}$ & $\mathrm{aOR}^{*}$ & $95 \% \mathrm{Ci}$ \\
\hline Low Birthweight $(<2500$ g) & 4.64 & $3.39-6.35$ & 4.79 & 3.48-6.59 \\
\hline Stillbirth & 3.68 & $2.42-5.62$ & 3.34 & 2.17-5.14 \\
\hline \multirow[t]{2}{*}{ Maternal death } & 4.93 & $1.22-19.89$ & 5.21 & $1.26-21.56$ \\
\hline & \multicolumn{4}{|c|}{ NT vs. EC } \\
\hline$\overline{\text { Outcomes }}$ & $\overline{\mathrm{OR}}$ & $95 \% \mathrm{CI}$ & $\mathbf{a O R}^{*}$ & $95 \% \mathrm{Ci}$ \\
\hline Low Birthweight $(<2500$ g) & 5.29 & $3.03-9.25$ & 5.00 & 2.84-8.79 \\
\hline Stillbirth & 5.78 & $3.16-10.57$ & 6.34 & $3.40-11.82$ \\
\hline Maternal death & 10.09 & 2.00-51.05 & 12.70 & 2.33-69.31 \\
\hline
\end{tabular}

ePOSS, Enhanced Routine Pregnancy Outcome Surveillance System; HDP, Hypertensive disorder in pregnancy; HAS, Hôpital Albert Schweitzer; NT, normotensive women; HTN, Hypertension; PEC, Preeclampsia; EC, Eclampsia; $\mathrm{OR}$, odds ratio; aRR, adjusted odds ratio

Statistically significant CI's are shown in bold.

*Adjusted for maternal age, parity, year of delivery

1743 pregnancies, and found that $16.6 \%$ (290) of women were diagnosed with preeclampsia and eclampsia, resulting in 48 stillbirths and 5 maternal deaths [9]. The current study used records from 2080 pregnancies and found the prevalence of HDP at HAS to be $13.5 \%$ (280) with 156 stillbirths and 11 maternal deaths. Raghuraman et al. looked at women who were diagnosed with antepartum preeclampsia, antepartum eclampsia, postpartum preeclampsia, and postpartum eclampsia, while the present study analyzed diagnoses of hypertension during pregnancy, preeclampsia, and eclampsia. Chart review and systematic data entry were used to assess maternal complications for both studies, and the resulting estimates of HDP prevalence are similar. Both studies found that there was no difference in maternal age among women with HDP and who had a stillbirth compared to women who had a live birth. More maternal deaths and placental abruptions were seen among the women with HDP and who had stillbirths in both studies. Significantly more stillbirths were identified in the current study, which authors believe is due to improved surveillance at HAS and the roll-out of the electronic data base.

One key difference between this study and the Raghuraman et al. study is the ability to compare women with HDP to women without HDP in this study. The Raghuraman study lacked medical data on women with no hypertensive disorders; thus, they were unable to have a group to compare complications and other associations [9]. 
The current study also examined HDP in three additional hospitals in other areas of Haiti, thereby allowing comparisons among different Departments and obtaining a broader perspective on hospital-based prevalence estimates. Prevalence of HDP varied among the four hospitals, and was much higher at HAS compared to the others. This could be due to HAS having a known electronic medical records system that has been implemented for many years; thus, they may have more extensive records and better record keeping. All hospitals are in rural areas; however, HAS is the largest referral hospital in Haiti in which they receive more complicated cases from other hospitals. The lack of consistency between the four hospitals may not only be differences among samples, but also differences in surveillance and record keeping. All hospitals, except Fort Liberté, found an association between HDP and higher proportions of low birth weight babies and stillbirths. The relative small number of women with HDP at HIC, Fort Liberté, and HSC may have masked some of the associations that were seen at HAS.

The limitations to this study include the retrospective nature of this hospital-based study. These findings are not generalizable to the Haitian population due to the large number of women who do not give birth inside a hospital or medical facility. This sample represents deliveries in these four hospitals in four Departments of Haiti.

Data were collected from existing surveillance records that had incomplete records and missing data. Gestational age was not collected in the surveillance system until July 2013, which affected records from HAS, Fort Liberté, and HSC. HIC outcomes were not recorded for women presenting at the OT, which may have caused associations to be missed since women who require surgical intervention often have more high-risk pregnancies and complications. Maternal deaths at HIC were poorly reported and appear to be differentially missing; women with reported HDP appear to be missing maternal vital status more than women without HDP. Data from Fort Liberté may not be representative of the population in that commune and District as this hospital does not have a surgery ward. Women who present at Fort Liberté with a high-risk pregnancy requiring surgery are referred out to other health facilities. A further limitation to this study is the inconsistency of years of data used for the various hospitals. It is expected that more years of implementing the surveillance system may be associated with more complete and accurate data reporting.

A limitation of this study is the lack of maternal variables that were being captured in ePOSS at the time of data analysis. History of chronic hypertension and diabetes mellitus were not able to be assessed as maternal outcome variables and known complications of preeclampsia and eclampsia, such as stroke or renal failure, were not able to be assessed as potential confounders. A further limitation of this study is the lack of specific blood pressure readings. Researchers were unable to confirm the readings; thus, there is a potential for misclassification of HDP. HDP and placental abruption complications are captured in the surveillance as three complication variables. Not having a specific recording section for each of these separately leads to a potential underreporting of these data. Heavier maternal weight, which has been shown to be associated with a 3-fold odds for HDP in a community-based sample of prenatal patients in Haiti, was also not recorded in this system [11]. In that study, maternal age of more than 40 years was also found to be associated with a 3-fold odds of HDP in a multivariable model that included maternal weight and the number of prenatal visits [11].

This study adds to the limited work studying HDP in LMIC's and in Haiti specifically. The lack of consistency between the four hospitals in this study exemplifies not only opportunities to improve surveillance in Haiti, as the true burden of HDP in this country remains unknown. Additional studies are needed to further examine the etiology of hypertension in Haitian women, especially during pregnancy. Due to the majority of Haitian mothers delivering outside of a medical facility, this large sample of the population remains unrepresented in statistics and in the literature. Qualitative and quantitative research at the community level can add to this research and allow for a comprehensive analysis of HDP among all Haitian mothers. Results from this study allows researchers to identify areas for further improvement at the hospital level and provides data that can aid in evidencebased intervention strategies. Studies like this one emphasizes the importance of detailed data collection and surveillance in order to better target investments, public health interventions, and public health policies.

\section{Conclusion}

In conclusion, the results of this study highlight the importance of HDP as a major cause of adverse maternal and neonatal outcomes among this population in Haiti, which is comparable to studies conducted in highincome countries. Specifically, women with eclampsia, the most severe form of HDP, had the worst health outcomes including an increased odds of having a low birth weight baby, delivering a stillbirth, and were more likely to die due to complications of pregnancy. More research is needed in Haiti to understand the true prevalence and impact of HDP in facility and home births.

\footnotetext{
Abbreviations

AOR: Adjusted Odds Ratio; BMI: Body Mass Index; CEmO: Comprehensive Emergency Obstetric Care Facilities; EMR: Electronic Medical Record; ePOSS: Enhanced Routine Pregnancy Outcome Surveillance System; HAS: Hôpital Albert Schweitzer; HDP: Hypertensive Disorders in Pregnancy; HIC: Hôpital Immaculée Conception des Cayes; HSC: Hôpital Sacré Coeur de Milot; LMIC: Low and middle-income countries; MDG: Millennium Development Goals; MMR: Maternal mortality ratio; NGO: Non-governmental
} 
organizations; OT: Operating Theater; SDG: Sustainable Development Goals; SOG: Soins Obstetricaux Gratuits

\section{Acknowledgements}

The project was a collaboration between Ministere de la Sante Publique et de la Population (MSPP), U.S. Centers for Disease Control and Prevention, and several hospitals and facilities throughout Haiti. The authors would like to thank the many people within these organizations who contributed to the success of this project.

\section{Authors' contributions}

All authors have read and approved the manuscript. EH, RGP, RLL, and DF prepared the study protocol; $\mathrm{EH}$ led the data collection; $\mathrm{MB}, \mathrm{EH}, \mathrm{MH}, \mathrm{CH}$, and $\mathrm{BP}$ analyzed the data; $\mathrm{MB}, \mathrm{EH}, \mathrm{MH}, \mathrm{CH}$, and $\mathrm{BP}$ wrote the manuscript. The findings and conclusions in this report are those of the authors and do not necessarily represent the official position of the Centers for Disease Control and Prevention.

\section{Funding}

The study was funded through cooperative agreements between CDC and MSPP Haiti, including several sub-contracts to the following partners: $\mathrm{PIH}$ CDS and Albert Schweitzer Hospital. CDC and MSPP Haiti developed the protocol. MSPP Haiti, along with their partners PIH, CDS, and Albert Schweitzer Hospital were responsible for data collection. CDC analyzed and interpreted the data. CDC wrote the manuscript and MSPP Haiti provided input and approved the manuscript.

\section{Availability of data and materials}

Data and material from which this manuscript was developed are available on reasonable request from the corresponding author.

\section{Ethics approval and consent to participate}

Ethical clearance for the wider study from which this paper is drawn was obtained from the U.S. Centers for Disease Control and Prevention. Protoco was reviewed and approved by the Haiti $\mathrm{MOH}$ and $\mathrm{CDC}$ determined the project was non-research; thus, IRB approval was not needed.

\section{Consent for publication}

Not applicable.

\section{Competing interests}

The authors declare that they have no competing interests. Both funding bodies (CDC and MSPP Haiti) were involved in the interpretation of the results and writing and approving the manuscript.

\section{Author details}

'U.S. Centers for Disease Control and Prevention, Atlanta, GA, USA. ${ }^{2}$ Department of Epidemiology, Rollins School of Public Health, Emory University, Atlanta, GA, USA. ${ }^{3}$ Ministère de la Santé Publique et de la Population, Port-au-Prince, Haiti. ${ }^{4}$ Hôpital Albert Schweitzer, Decapelle, Haiti.

Received: 21 March 2019 Accepted: 13 June 2019

Published online: 20 June 2019

\section{References}

1. World Health Organization. Trends in maternal mortality: 1990 to 2015. 2015. Retrieved from http://apps.who.int/iris/bitstream/10665/193994/1/ WHO_RHR_15.23_eng.pdf?ua=1.

2. World Health Orgnaization; UNICEF. Stillbirths: the invisible public health problem. 2011. Retrieved from http://www.who.int/pmnch/media/news/ 2011/20110414_stillbirths_pressrelease.pdf.

3. World Health Organization. Maternal mortality. 2016. Retrieved from http:// www.who.int/mediacentre/factsheets/fs348/en/

4. World Health Organization. Haiti. 2016. Retrieved from http://www who.int/countries/hti/en/

5. Jiao J e a. Hypertension prevalence: an examination of urban and rural Haiti. Lancet Glob Health. 2014;2:S25

6. Dickstein Y, Neuberger A, Golus M, Schwartz E. Epidemiologic profile of patients seen in primary care clinics in an urban and a rural setting in Haiti, 2010-11. Int Health. 2014;6(3):258-62. https://doi.org/ 10.1093/inthealth/ihu033.
7. Jean-Baptiste ED, Larco P, Charles-Larco N, Vilgrain C, Simon D, Charles R. Glucose intolerance and other cardiovascular risk factors in Haiti. Prevalence of diabetes and hypertension in Haiti (PREDIAH). Diabetes Metab. 2006:32(5 Pt 1):443-51.

8. Handzel EW, Grand-Pierre R, Louis RJ, De Jesus S, Felker-Kantor E, et al. Pregnancy Outcome Surveillance System (POSS): Standard Operating Procedures. Centers for Disease Control and Prevention. 2014.

9. Raghuraman N, March MI, Hacker MR, Modest AM, Wenger J, Narcisse R, et al. Adverse maternal and fetal outcomes and deaths related to preeclampsia and eclampsia in Haiti. Pregnancy Hypertension. 2014;4(4): 279-86. https://doi.org/10.1016/j.preghy.2014.09.002.

10. Small MJ, Kershaw T, Frederic R, Blanc C, Neale D, Copel J, Williams KP. Characteristics of preeclampsia- and eclampsia-related maternal death in rural Haiti. Journal of Maternal-Fetal \& Neonatal Medicine. 2005;18(5):343-8. https://doi.org/10.1080/14767050500312433.

11. Sekkarie A, Raskind-Hood C, Hogue C. The effects of maternal weight and age on pre-eclampsia and eclampsia in Haiti. Journal of Maternal-Fetal \& Neonatal Medicine. 2015:1-5. https://doi.org/10.3109/14767058.2015. 1012064.

12. République d'Haiti Ministére de la Santé Publique et de la Population (MSPP). Protocoles de prise en charge des complications obstétricales et néonatales. 2015.

13. World Health Organization. WHO recommendations for prevention and treatment of pre-eclampsia and eclampsia. 2011. Retreived from https:// www.who.int/reproductivehealth/publications/maternal_perinatal_health/ 9789241548335/en/.

14. Sibai B, Dekker G, Kupferminc M. Pre-eclampsia. Lancet. 2005;365(9461):78599. https://doi.org/10.1016/s0140-6736(05)17987-2.

15. Moroz LA, Simpson LL, Rochelson B. Management of severe hypertension in pregnancy. Semin Perinatol. 2015. https:/doi.org/10.1053/.j.semperi.2015.11.017.

16. Cunningham FG, e. a. Williams Obstetrics (Vol. 24th edition): McGraw-hill Education; 2014

\section{Publisher's Note}

Springer Nature remains neutral with regard to jurisdictional claims in published maps and institutional affiliations.

\section{Ready to submit your research? Choose BMC and benefit from:}

- fast, convenient online submission

- thorough peer review by experienced researchers in your field

- rapid publication on acceptance

- support for research data, including large and complex data types

- gold Open Access which fosters wider collaboration and increased citations

- maximum visibility for your research: over $100 \mathrm{M}$ website views per year

At BMC, research is always in progress.

Learn more biomedcentral.com/submissions 